\title{
Three Distinct Amine Receptors Operating at Different Levels within the Locomotory Circuit Are Each Essential for the Serotonergic Modulation of Chemosensation in Caenorhabditis elegans
}

\author{
Gareth P. Harris, Vera M. Hapiak, Rachel T. Wragg, Sarah B. Miller, Lindsay J. Hughes, Robert J. Hobson, \\ Robert Steven, Bruce Bamber, and Richard W. Komuniecki \\ Department of Biological Sciences, University of Toledo, Toledo, Ohio 43606
}

\begin{abstract}
Serotonin modulates behavioral plasticity in both vertebrates and invertebrates and in Caenorhabditis elegans regulates key behaviors, including locomotion, aversive learning and olfaction through at least four different 5-HT receptors. In the present study, we examined the serotonergic stimulation of aversive responses to dilute octanol in animals containing null alleles of these 5-HT receptors. Both ser-1 and mod-1 null animals failed to increase sensitivity to dilute octanol on food/5-HT, in contrast to wild-type, ser-4 or ser-7 null animals. 5-HT sensitivity was restored by the expression of MOD-1 and SER-1 in the AIB or potentially the AIY, and RIA interneurons of mod-1 and ser-1 null animals, respectively. Because none of these 5-HT receptors appear to be expressed in the ASH sensory neurons mediating octanol sensitivity, we identified a 5-HT 6 -like receptor, F16D3.7(SER-5), that was required for food/5-HT-dependent increases in octanol sensitivity. ser-5 null animals failed to increase octanol sensitivity in the presence of food/5-HT and sensitivity could be restored by expression of SER-5 in the ASHs. Similarly, the RNAi knockdown of ser-5 expression in the ASHs of wild-type animals also abolished 5-HT-dependent increases in octanol sensitivity, suggesting that SER-5 modulates the octanol responsiveness of the ASHs directly. Together, these results suggest that multiple amine receptors, functioning at different levels within the locomotory circuit, are each essential for the serotonergic modulation of ASH-mediated aversive responses.
\end{abstract}

Key words: ASH neuron; G-protein-coupled receptors; locomotion; plasticity; reversals; serotonin (5-HT)

\section{Introduction}

Behavioral plasticity is often mediated by biogenic amines (BAs), such as serotonin (5-HT). In mammals, 5-HT regulates diverse central and peripheral responses, including circadian rhythms, mood, perception and aggression and a variety of drugs modulate serotonergic signaling in the treatment of schizophrenia, depression and migraine (Lucki, 1998; Heisler et al., 2007). Changes in serotonergic signaling can have profound effects on behavior. However, although all of the mammalian 5-HT receptors have been cloned and characterized, much still remains to be learned about how they are integrated into complex multilevel signaling pathways. Therefore, this study was designed to identify and localize the Caenorhabditis elegans 5-HT receptors involved in the modulation of a simple, sensory-mediated, locomotory behavior, i.e., aversive responses to the volatile repellant, octanol.

Received Sept. 23, 2008; revised Dec. 11, 2008; accepted Dec. 14, 2008.

This work was supported by National Institutes of Health Grant Al-145147, awarded to R.W.K., and funds from the Joan L. and Julius H. Jacobson Biomedical Professorship. We thank Dr. Shohei Mitani at the National Bioresources Project, Drs. Cori Bargmann, Gert Jansen, Yuji Kohara, Villu Maricq, Ji Sze, and the Caenorhabditis Genetics Center for strains, and Dr. Anne Hart for critically reading portions of this manuscript.

Correspondence should be addressed to Richard W. Komuniecki, Department of Biological Sciences, University of Toledo, 2801 West Bancroft Street, Toledo, OH 43606-3390. E-mail: rkomuni@uoft02.utoledo.edu.

DOI:10.1523/JNEUROSCI.4585-08.2009

Copyright @ 2009 Society for Neuroscience $\quad$ 0270-6474/09/291446-11\$15.00/0
C. elegans has a simple nervous system (302 neurons) and can detect environmental stimuli through 14 pairs of specialized sensory neurons. More importantly, many sensory-mediated aversive responses are modulated by 5 -HT, making C. elegans an excellent model to study the aminergic modulation of sensorymediated locomotory behaviors. 5-HT is a "food is at hand" signal for starved animals and modulates most key behaviors, including aversive learning, egg-laying, feeding and locomotion, supporting the suggestion that 5 -HT levels communicate nutritional state (Horvitz et al., 1982; Segalat et al., 1995; Waggoner et al., 1998; Sawin et al., 2000; Niacaris and Avery, 2003; Zhang et al., 2005). The polymodal ASH sensory neurons are located in specialized amphidial structures in the head and are essential for the avoidance of a variety of aversive stimuli, including both volatile repellants and mechanical stimuli, such as nose touch (de Bono and Marique, 2005). For example, the ASH neurons are necessary and sufficient for the detection of dilute octanol, and 5-HT (or the presence of food) potentiates ASH-mediated responses, i.e., sensitivity to dilute octanol is increased in the presence of 5-HT or when the animals are on food (Troemel et al., 1997; Chao et al., 2004). In addition, $\mathrm{Ca}^{2+}$ transients in the ASHs can be detected in response to nose touch only if 5-HT is present, suggesting that the ASHs are modulated directly to 5-HT (Hilliard et al., 2005). 
In the present study, we identified a subset of amine receptors that are each essential for 5-HT-dependent increases in aversive responses to dilute octanol. These 5-HT receptors appear to operate at different levels within the nervous system to integrate sensory input with locomotory behavior, i.e., SER-5 in the ASH sensory neurons, MOD-1 in the AIB, and possibly AIY, interneurons that integrate signals directly from the ASH sensory neurons and SER-1 in the RIA interneurons, major upstream partners of the ring motor neurons that innervate head muscle. Based on the comprehensive wiring diagram of the C. elegans nervous system, we predict that SER-5 increases the responsiveness of the ASHs to dilute octanol, MOD-1 modulates interneuronal signaling to stimulate reversal and backward locomotion and SER-1 is potentially involved in the control of head muscle. These observations highlight the complexity of serotonergic modulation and the advantages of the $C$. elegans model for studying the modulation of amine-mediated chemosensory circuits.

\section{Materials and Methods}

Materials. All reagents were purchased from Sigma-Aldrich. DMEM was purchased from Mediatech, neurochemicals from Sigma-Aldrich, restriction enzymes from New England Biolabs, and Promega, and oligonucleotide primers from Integrated DNA Technologies. A C. elegans cDNA pool was purchased from OriGene Technologies, and additional cDNA pools were constructed from mixed stage mRNA using standard techniques. Green fluorescent protein (GFP) expression vectors were obtained from Andy Fire (Stanford University School of Medicine, Stanford, CA).

Cultures and maintenance of strains. The N2 Bristol WT isolate of $C$. elegans was used for all studies. All animals were raised at $20^{\circ} \mathrm{C}$ under uncrowded conditions (Brenner, 1974). The following putative 5-HT receptor null alleles were used in this study: ser-5 (tm2647)I, ser5(tm2654)I, ser-4(ok512)III, mod-1(ok103)V, ser-7(tm1325)X, ser1 (ok345)X. In addition, the following strains were also examined, $r r f$ 3 (pk1269) and $t t x-3(k s 5)$. All strains were obtained from the Caenorhabditis Genetics Center (University of Minnesota, Minneapolis, MN) except ser-7(tm1325), ser-5(tm2647) and ser-5(tm2654) which were received from the National Bio-Resources Project (Tokyo Women's Medical University, Tokyo, Japan).tph-1 (mg280) animals were provided by Dr. Mark Alkema (Massachusetts Institute of Technology, Boston, MA), $t t x-3 p:: m o d-1$ and $o d r-2(2 b) p::$ mod-1 animals by Dr. Cori Bargmann (Rockefeller University, New York, NY) (Zhang et al., 2005), ser1(ok345); mod-1(ok103) animals from Dr. Ji Ying Sze (Albert Einstein College of Medicine, New York, NY) and glr-3p::ICE animals from Dr. Villu Maricq (University of Utah, Salt Lake City, UT). Animals containing combinations of 5-HT receptor null alleles were constructed using standard genetic techniques and confirmed by PCR. All mutant animals were backcrossed with the N2 Bristol strain at least five times before use.

Behavioral assays. Assay plates [ $5 \mathrm{~cm}$ Nematode growth medium plates (NGM)] were prepared daily and serotonin (4 mM) was added to NGM liquid media just before pouring. Dilute 1-octanol was prepared daily using 100\% ethanol (v/v) (Sulston and Hodgkin, 1988; Bargmann et al., 1993; Chao et al., 2004). Synchronized fourth-stage larvae (L4) were picked $24 \mathrm{~h}$ preassay and assays were performed at $23-25^{\circ} \mathrm{C}$. Octanol avoidance was measured as described by Chao et al. (2004). Briefly, the blunt end of a hair (Loew-Cornell 9000 Kolinsky 8 paintbrush), taped to a toothpick, was dipped in $30 \%$ octanol and the hair was placed in front of an animal exhibiting forward sinusoidal locomotion. Time to reverse was recorded and assays were terminated after $20 \mathrm{~s}$, because wild-type animals spontaneously reverse on average every 20 s (Zhao et al., 2003; Chao et al., 2004, 2005). For assays in the absence of food or exogenous 5 -HT, well fed young adults (three to five per plate) were first transferred to intermediate nonseeded plates and left for $1 \mathrm{~min}$ to prevent any bacteria/media carry over, then transferred to NGM plates and assayed after $10 \mathrm{~min}$. For assays in the presence of food (E. coli OP50) or 5-HT, animals were transferred to plates containing a thin layer of OP50 or 4 mM 5-HT and assayed after 20 and $30 \mathrm{~min}$, respectively.
Reversal frequency was assayed as described previously (Tsalik and Hobert, 2003; Dernovici et al., 2006). Well-fed animals were transferred to NGM plates for $30 \mathrm{~s}$, then transferred to assay plates ( \pm food) for $1 \mathrm{~min}$ and assayed. Reversal frequency was scored as the number of times an animal reversed within $3 \mathrm{~min}$ (Pierce-Shimomura et al., 1999). Data were presented as a mean \pm SE $(n=3)$ and analyzed by two-tailed Student's $t$ test. $p$ values were indicated as follows: ${ }^{\star} p<0.05,{ }^{* *} p<0.01,{ }^{\star * *} p<$ 0.001 .

RNA interference. RNA interference (RNAi) was performed as described previously using wild-type N2 and $r r f-3(p k 1629)$ animals (Kamath and Ahringer, 2003). Hermaphrodites and their progeny were grown on NGM plates containing $25 \mu \mathrm{g}$ of carbenicillin and $1 \mathrm{~mm}$ isopropyl- $\beta$-D-thiogalactopyranosidase (IPTG) and were seeded with HT115 (DE3) bacteria containing either the ser-5 RNAi vector or empty vector. Synchronized L4s were picked $24 \mathrm{~h}$ preassay and examined for octanol sensitivity.

Rescue constructs and strains. All rescue constructs were created by overlap fusion PCR or by cloning into pPD95.75 (Mello and Fire, 1995; Hobert, 2002). For overlap PCR, constructs were pooled from at least 3 reactions and were coinjected with $m y o-3 p:: g f p, F 25 B 3.3 p:: g f p$ or rol-6 and carrier DNA (to $100 \mathrm{ng}$ ) into gonads of wild-type and null mutant animals by standard techniques (Kramer et al., 1990; Mello and Fire, 1995). At least three lines expressing each construct were examined. All constructs in pPD95.75 were confirmed by sequencing.

FY748 mod-1(ok103)grEx160[mod-1p::mod-1] expresses a fulllength mod-1 transgene in mod-1 null animals. The mod-1 transgene includes a $2 \mathrm{~kb}$ mod-1 promoter, the mod-1 open reading frame and $706 \mathrm{bp}$ of the mod-1 3'UTR, and was created with MOD-1F1 and MOD-1R1, as described by (Carnell et al., 2005). FY751 ser-5(tm2654)grEx163[sra-6p::ser-5::gfp] expresses SER-5 in the ASH sensory neurons of ser-5 null animals. The sra-6p::ser-5::gfp transgene includes the sra- 6 promoter, the ser- 5 cDNA, sequence coding for GFP and the $u n c-543^{\prime} \mathrm{UTR}$. The $3.4 \mathrm{~kb}$ sra- 6 promoter was amplified with SRA-6F1 and SRA-6R+SER-51 and the ser-5 cDNA with SER$5 \mathrm{~F} 2$ and SER-5R+GFP2. The sra- 6 promoter was fused to the ser-5 cDNA using nested primers, SRA-6F3 and SER-5R3. $g f p$ including the unc-54 3'UTR was amplified from pPD95.75 with GFPF4a and GFPR4 and the sra-6p::ser-5 product was then fused to $g f p$ using nested primers, SRA6F5 and GFPR5. FY750 ser-5(tm2654)grEx162[ser-5p::ser-5] expresses a fulllength ser-5 transgene in ser-5 null animals. The ser-5p::ser-5 transgene with a $5 \mathrm{~kb}$ ser- 5 promoter was amplified with SER-5F1 and SER-5R1 to generate an $8.6 \mathrm{~kb}$ product. RWK8 mod-1 (ok103)fvEx3[npr-9p::mod-1::gfp] expresses MOD-1 in the AIB interneurons of mod-1 null animals. The $n p r-9 p::$ mod-1::gfp transgene includes $2 \mathrm{~kb}$ of the $n p r-9$ promoter, the mod-1 cDNA, sequence coding for GFP and the unc-54 3' UTR. The npr-9 promoter was amplified with NPR-9F1 and NPR-9R+MOD-11, the mod-1 CDNA with MOD-1F2 and MOD-1R2 and $g f p$ with the primers described above, except that the $g f p$ forward primer contained an overlapping fragment of the 3 ' end of the mod-1 cDNA (GFPF+MOD-13). $n p r-9 p$ was fused to the mod-1 cDNA with NPR-9F4 and MOD-1R4 and npr-9p::mod-1 was fused to $g p$ with NPR-9F5 and GFPR5.

Localization of SER-5 expression. RWK7 ser-5(tm2654)fvEx2[ser-5p::ser-5::gfp] expresses a full-length ser-5 translational fusion from the $5 \mathrm{~kb}$ ser- 5 promoter that includes the endogenous ser $-53^{\prime}$ UTR with sequence encoding GFP inserted into the predicted SER-5 C terminus 10 aa after TMVII. The $s e r-5:: g f p$ transgene was created by overlap fusion PCR from three different products fused by two rounds of PCR. Primers amplified the ser- $55^{\prime}$-end $\sim 5 \mathrm{~kb}$ upstream of the ATG up to a portion of exon 6 using SER-5F1 and SER-5R+GFP1. A second set of primers (SER$5 F+$ GFP2 and SER-5R2) amplified a short $g f p$ overlap with the ser-5 3' end including the predicted SER-5 C terminus 10 aa after TMVII and the ser-5 $3^{\prime} \mathrm{UTR}$, as follows. Sequence encoding $g f p$ was amplified using GFPF3b and GFPR 3 and then fused in frame with the ser-5 $3^{\prime}$ product (using GFPF4 and SER-5R4) and this product was then combined with the ser-5 5' fragment to generate a full-length ser-5 transgene that included sequence coding for GFP inserted in the predicted C terminus of the receptor using nested primers. PCR products were pooled from at least 3 separate reactions ( $5 \mathrm{ng}$ total) and were coinjected either alone or with rol-6 plasmid (pRF4) and carrier DNA into gonads of ser-5(tm2564) 
null animals by standard techniques (Mello and Fire, 1995). Uptake of DiD in living animals was assayed as described (Herman and Hedgecock, 1990). Briefly, stock solution (1 mM) of 1,1'-dioctadecyl-3,3,3,3'tetrametylindocarbocyanine (DiD) (Molecular Probes/Invitrogen Labeling and Detection) was diluted 1:200 in M9 buffer. Animals were incubated in $100 \mu \mathrm{l}$ of diluted $\mathrm{DiD}$ for $1 \mathrm{~h}$ at RT, transferred to a fresh NGM plate seeded with OP50 and allowed to crawl on the bacterial lawn for $1-2 \mathrm{~h}$ to destain and were then placed on agarose pads with $20 \mathrm{~mm}$ sodium azide for visualization. At least five transformed lines were analyzed for $g f p$ fluorescence and DiD staining using an Olympus confocal microscope.

Cell-specific RNAi knockdown. Transgenes for cell-specific knockdown were constructed as described (Esposito et al., 2007). RWK12 $f v E x 7$ [sra-6p::ser-5RNAi] expressed a ser-5(RNAi) in the ASH sensory neurons of wild-type animals. The sra-6p::ser-5(RNAi) transgene included the sra- 6 promoter fused to exon rich regions of ser-5. The sra- 6 promoter was amplified with SRA-6PF1 and the reverse (sense) primer SRA-6PRS1 and the reverse (antisense) primer SRA-6PRA2 to create templates $\mathrm{B}$ and $\mathrm{C}$, respectively. The ser- 5 coding region was amplified from genomic DNA using SER-5TF2 and SER-5TR4 to create template A. Templates A and B were fused using SRA-6F3 and the reverse nested primer SER-5R5 to create the sense construct. Templates A and C were fused using SRA-6F4 and SER-5TF6 to create the antisense construct. RWK13 fvEx6[glr-3::ser-1RNAi] expressed a ser-1(RNAi) in the RIA interneurons. The $g l r-3 p:: s e r-1(R N A i)$ transgene included the $g l r-3$ promoter fused to exon rich regions of ser- 1 . The $g l r-3$ promoter was amplified with GLR-3PF1 and the reverse (sense) primer GLR-3PRS1 and the reverse (antisense) primer GLR-3PRA2 to create templates B and C, respectively. The ser- 1 coding region was amplified from genomic DNA using SER-1TF2 and SER-1TR3 to create template A. Templates A and B were fused using GLR-3F3 and SER-1TR4 to create the sense construct. Templates A and C were fused using GLR-3F4 and SER-1TF5 to create the antisense construct. Sense and antisense transgenes were both microinjected at $100 \mathrm{ng} / \mu \mathrm{l}$ (a list of sense and antisense primers used for the generation of PCR fusions is included in supplemental material, available at www.jneurosci.org as supplemental material).

Heterologous expression of SER-5. To express SER-5, pFLAG-SER-5 or pDisplay-SER-5 were transiently transfected into COS-7 cells using Lipofectamine 2000 (Invitrogen; $1 \mu \mathrm{g}$ of DNA/3 $\mu \mathrm{l}$ of Lipofectamine), as we have described for other nematode GPCRs (Huang et al., 2002; Hobson et al., 2003). Cells were harvested $48 \mathrm{~h}$ post-transfection and membranes were prepared as described previously (Rex and Komuniecki, 2002). Briefly, COS-7 cells were washed with ice-cold PBS and incubated in $15 \mathrm{~mm}$ Tris- $\mathrm{HCl}, \mathrm{pH} 7.4,1 \mathrm{~mm}$ EDTA and $1 \mathrm{~mm}$ phenylmethylsulfonylfluoride (PMSF) at $4^{\circ} \mathrm{C}$ for $10 \mathrm{~min}$. Cells were scraped from plates, centrifuged at $4000 \times \mathrm{g}$ for $15 \mathrm{~min}$ and pellets resuspended in ice-cold TEM (Tris- $\mathrm{HCl} \mathrm{pH} \mathrm{7.4,} 10 \mathrm{~mm} \mathrm{MgCl}_{2}, 0.5 \mathrm{~mm}$ EDTA) containing $1 \mathrm{~mm}$ PMSF. Cells were lysed on ice by sonication, centrifuged at $20,000 \times g$ for $1 \mathrm{~h}$ at $4^{\circ} \mathrm{C}$ and the pellet homogenized in TEM buffer. Radioligand binding was performed as previously described (Huang et al., 2002; Hobson et al., 2003).

Multiple sequence alignments and production of phylogenetic trees. To remove hypervariable regions, each vertebrate and invertebrate 5-HT receptor sequence was truncated at the $\mathrm{N}$ terminus (three residues before TMI), the third intracellular loop (eight residues after TMV and six residues before TMVI), and the C terminus (12 residues after TMVII). The initial alignment was performed with the modified sequences using the MegAlign program (DNAStar) with ClustalW parameters set to optimize protein sequence alignments (multiple alignment parameters: Gap opening penalty of 15 , and gap extension penalty of 0.3 ; pairwise alignment parameters: Gap opening penalty of 35 , and gap extension penalty of 0.75 ). The resulting alignment was then fine-tuned manually. All alignments are available on request. Bootstrapping was also performed with DNAStar (1000 replicates, random seed), and the resulting tree was then imported into the Phylogenic Alignment Utility Program (PAUP, version 4.0b10), where it was analyzed under the distance, neighbor-joining method to produce the tree reported here. The Caenorhabditis elegans GAR-1 sequence was used as an outgroup. Accession numbers for all sequences used are listed in the legend to Figure 5.

\section{Results}

\section{SER-1 and MOD-1 are each essential in modulating} sensitivity to dilute octanol

Wild-type animals on food (E. coli OP50) or incubated in exogenous 5-HT (4 mM) exhibit dramatically increased aversive responses to dilute octanol, but the serotonergic receptors and pathways mediating these effects have not been identified (Chao et al., 2004). C. elegans contains at least four previously characterized 5-HT receptors: three G-protein-coupled receptors, SER-1, SER-4 and SER-7, and a novel 5-HT-gated $\mathrm{Cl}^{-}$channel, MOD-1 (Olde and McCombie, 1997; Hamdan et al., 1999; Ranganathan et al., 2000; Hobson et al., 2003). Animals carrying null alleles for each of these 5-HT receptors have been characterized in other behavioral contexts (Ranganathan et al., 2000; Hobson et al., 2003; Dempsey et al., 2005; Zhang et al., 2005). To identify the individual 5-HT receptor(s) and neurons involved in the serotonergic modulation of octanol avoidance, we examined the responses of each of these 5-HT receptor null mutants to dilute (30\%) octanol, a behavior mediated by the ASH sensory neurons (Chao et al., 2004). The responses of these mutant animals to dilute octanol were identical to those of wild-type animals either off food or in the absence of exogenous 5-HT (Fig. 1). However, both the ser-1 and mod-1 null animals failed to increase their sensitivity to dilute octanol on food or in the presence of exogenous 5-HT, in contrast to ser-7, ser-4 or wild-type animals (Fig. 1). The role of mod-1 and ser-1 in 5-HT sensitivity was confirmed by transgenic rescue, i.e., the expression of full-length mod-1 or ser- 1 transgenes in mod-1 and ser- 1 null animals, respectively, restored 5-HT sensitivity (Figs. 2A, 3A). As predicted, ser-4; mod-1; ser-7 ser-1 quadruple null animals that lack all previously identified 5-HT receptors also failed to increase sensitivity to dilute octanol in the presence of either food or 5-HT (Fig. 1). These results suggest that both ser-1 and mod-1, but not ser- 4 or ser-7, have essential roles in the modulation of food- and 5-HTdependent increases in sensitivity to dilute octanol.

\section{MOD-1 and SER-1 function in the AIB or AIY and the RIA interneurons, respectively, to modulate ASH-mediated aversive responses to dilute octanol}

The neuronal expression of mod-1 and ser- 1 has been characterized previously using various mod-1p::gfp and ser-1p::gfp reporter constructs. mod-1p::gfp transcriptional fusions are expressed in the AIA, AIB, AIY, AIZ, and RID interneurons, as well as a number of additional unidentified neurons in the head, ventral cord and tail. Although ser-1p::gfp constructs depending on the ser-1::gfp transgene exhibit variable expression, in pharyngeal and vulval muscles, as well as a number of neurons in the head and tail, including the RIA interneurons and the ring and ventral cord motor neurons (Ranganathan et al., 2000; Tsalik et al., 2003; Wenick and Hobert, 2004; Carnell et al., 2005; Dempsey et al., 2005; Xiao et al., 2006; Dernovici et al., 2007).

To identify the neurons involved in the MOD-1-mediated modulation of ASH-mediated aversive responses, we examined the 5-HT sensitivity of aversive responses to dilute octanol in mod-1 null animals expressing $t$ tx-3p::mod-1 or $o d r-2(2 b) p::$ mod -1 transgenes previously observed to rescue the 5 -HT sensitivity of aversive olfactory learning in mod-1 null animals (Zhang et al., 2005). The $t+x-3$ promoter appears to be expressed exclusively in the AIY interneurons in adult animals, whereas $o d r-2(2 b) p$ drives expression primarily in the AIB and AIZ interneurons (Hobert et al., 1997; Chou et al., 2001; Wenick and Hobert, 2004; Bendena et al., 2008). In addition, we also examined mod-1 null animals expressing an npr-9p::mod-1 trans- 


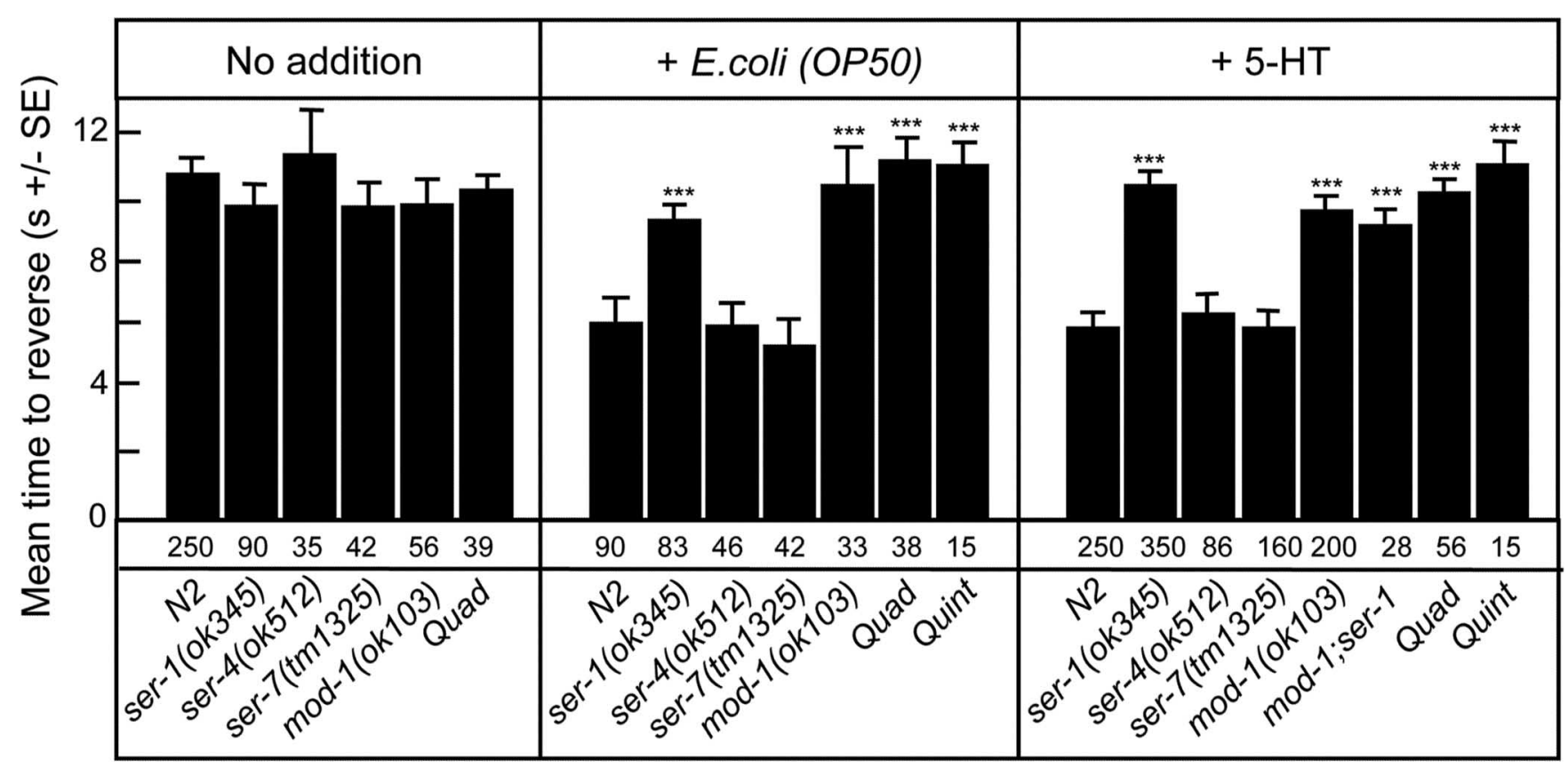

Figure 1. Food- and 5-HT-dependent increases in sensitivity to dilute octanol require both MOD-1 and SER-1. Wild-type and 5-HT receptor null mutant animals were examined for their ability to respond to dilute (30\%) octanol in the presence or absence of food (E. coli OP50) or 5-HT (4 mM), as described in Materials and Methods. Data are presented as mean \pm SE and analyzed by a two-tailed Student's $t$ test. ${ }^{* *} p<0.001$; significantly different from wild-type animals under identical test conditions. Numbers of trials are indicated under the bars. Quad, ser-4(ok512); mod-1(ok103); ser-7(tm1325) ser-1(ok345); Quint, ser-5(tm2654); ser-4(ok512); mod-1(ok103); ser-7(tm1325) ser-1(ok345).

A. Dilute octanol $(+5-\mathrm{HT})$

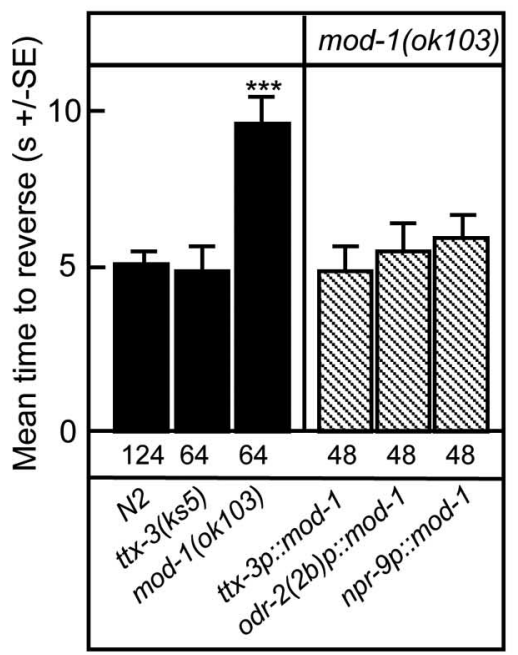

B. Spontaneous reversals (off food)

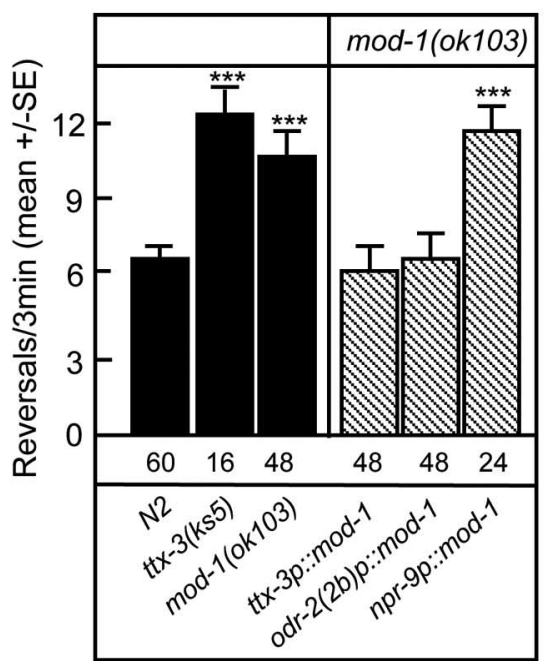

C. Spontaneous reversals (on food)

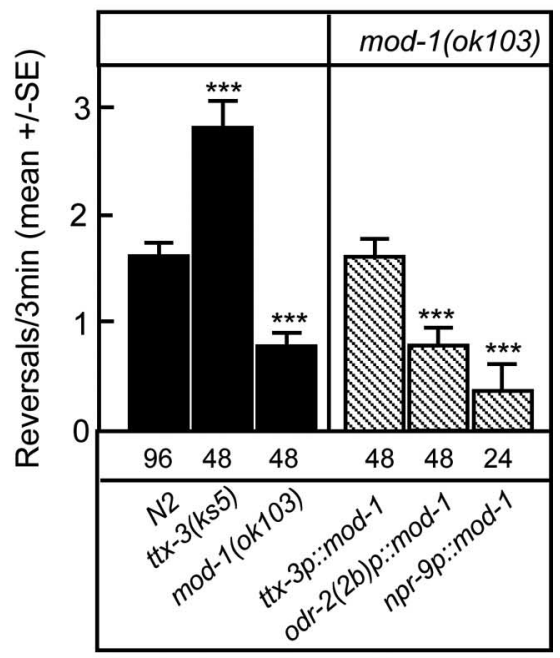

Figure 2. Rescue of 5 -HT-dependent increases in aversive responses to dilute octanol and food-dependent alterations in the rate of spontaneous reversals in mod- 1 null animals. $A$, Wild-type or mutant animals expressing the indicated transgenes were assayed for 5 -HT-dependent increases in aversive responses to dilute (30\%) octanol, as described in Materials and Methods. $\boldsymbol{B}$, $\boldsymbol{C}$, Spontaneous reversals (reversals $/ 3 \mathrm{~min}$ ) were quantified in well fed animals $1 \mathrm{~min}$ after transfer to plates without $(\boldsymbol{B})$ or with $(\boldsymbol{C})$ E. coli OP50. Data are presented as mean \pm SE and analyzed by a two-tailed Student's $t$ test. ${ }^{* *} p<0.001$, significantly different from wild-type animals under identical test conditions. The number of trials is indicated under the bars, and results from rescued mod -1 null animals are indicated by hatched bars.

gene that is expressed almost exclusively in the AIB interneurons (Bendena et al., 2008). All three transgenes restored 5-HT sensitivity in mod-1 null animals (Fig. $2 A$ ). Interestingly, ttx-3 null animals in which the AIYs fail to develop exhibited wild-type responses to dilute octanol both on and off food (Hobert et al., 1997) (Fig. 2A). However, because mod-1 encodes an inhibitory $\mathrm{Cl}^{-}$channel, animals that lack AIYs, such as the $t+x-3$ null animals or animals in which the AIYs are inhibited, as in the mod-1 expressing animals, would both be predicted to increase aversive responses to dilute octanol in the presence of food or 5-HT, exactly as we observed in the present studies. Together, these results suggest that the AIB and AIY interneurons are involved in modulating the 5-HT sensitivity to dilute octanol.

As noted above, the 5-HT sensitivity of ser- 1 null animals was rescued with a full-length ser-1p::ser-1::gfp transgene (Fig. 3A). This ser-1p::ser-1::gfp transgene contains a truncated $3.6 \mathrm{~kb}$ promoter and is expressed in the RIA interneurons and many of the ring motor neurons, major downstream partners of the RIAs, but 
A. Dilute octanol $(+5-\mathrm{HT})$

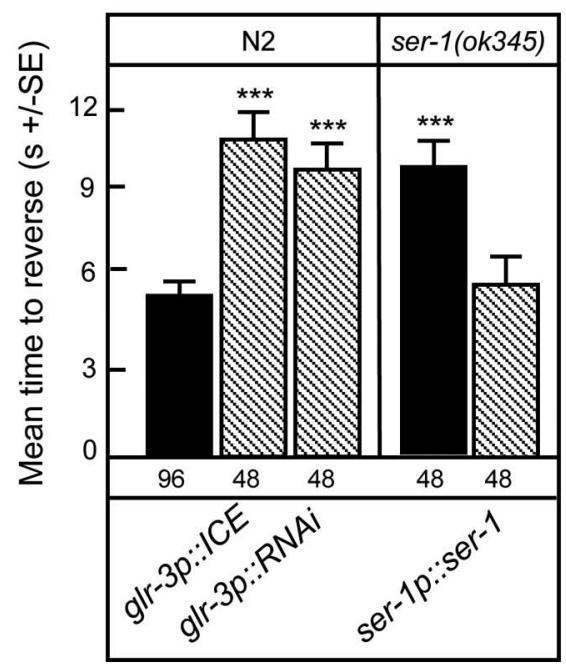

B. Spontaneous reversals (off food)

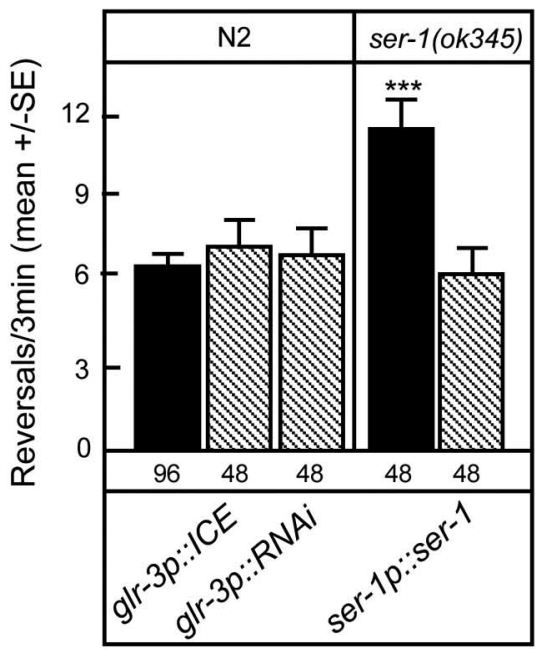

\section{Spontaneous reversals (on food)}

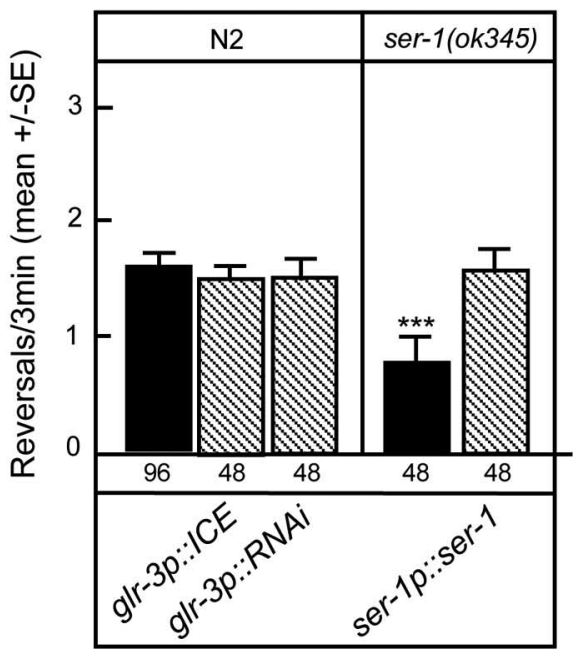

Figure 3. Rescue of 5-HT-dependent increases in aversive responses to dilute octanol and food-dependent alterations in the rate of spontaneous reversals in ser-1 null animals. $\boldsymbol{A}$, Wild-type or mutant animals expressing the indicated transgenes were assayed for 5-HT-dependent increases in aversive responses to dilute (30\%) octanol as described in Materials and Methods. $\boldsymbol{B}$, $\mathbf{C}$, Spontaneous reversals (reversals/3 min) were quantified in well fed animals $1 \mathrm{~min}$ after transfer to plates without $(\boldsymbol{B})$ or with $(\boldsymbol{C})$ E. coli OP50. Data are presented as mean \pm SE and analyzed by a two-tailed Student's $t$ test. ${ }^{* * *} p<0.001$, significantly different from wild-type animals under identical test conditions. The number of trials is indicated under the bars, and results from rescued ser- 1 null animals are indicated by hatched bars.

apparently not in the ventral cord motor neurons, as noted for other ser-1p::gfp transgenes (Carnell et al., 2005; Xiao et al., 2006; Dernovici et al., 2007). Therefore, because the ASHs innervate the RIAs directly, we examined the potential role of this RIAs/ring motor neuron pathway in the modulation of serotonergic signaling mediating aversive behavior. However, our attempts to rescue ser-1 null animals with a glr-3p::ser-1 transgene were unsuccessful, most probably because the over-expression of ser- 1 in the RIAs from the $g l r-3$ promoter alters RIA morphology. It has been our experience that neuronal development can be sensitive to the over-expression of some $\mathrm{G}_{\mathrm{s}}$ - and $\mathrm{G}_{\mathrm{q}}$-coupled amine receptors (Hobson et al., 2003, R. W. Komuniecki, unpublished observation). Therefore, we instead examined wild-type animals expressing either a $g l r-3 p:: I C E$ transgene that selectively ablates the RIAs or a $g l r-3 p:: s e r-1(R N A i)$ transgene that should specifically knockdown ser-1 expression in the RIAs (Fig. 3A). Animals expressing either transgene exhibited wild-type responses to dilute octanol off food, suggesting that the pathways sensing dilute octanol were intact (data not shown). In contrast, both animals failed to increase octanol sensitivity when incubated in 5-HT, further supporting a role for the RIAs in the SER-1-mediated serotonergic modulation of olfaction (Fig. $3 A$ ).

Together, these results suggest that mod-1 functions in the $\mathrm{AIB}$ and AIY interneurons and ser- 1 functions in the RIA/ring motor neuron pathway that ultimately innervates primarily head muscle in the serotonergic sensitization of aversive responses to dilute octanol (White et al., 1986; Alkema et al., 2005).

\section{The expression of SER-5 in the ASH sensory neurons is essential for the serotonergic modulation of aversive responses to dilute octanol}

As noted previously, the ASH sensory neurons are necessary and sufficient for responses to dilute octanol and 5-HT increases calcium transients in the ASH sensory neurons, suggesting that a 5-HT receptor may be expressed directly in the ASHs (Chao et al., 2004; Hilliard et al., 2005). However, none of the previously characterized 5-HT receptors appears to be expressed in the ASHs, suggesting that an additional $5-\mathrm{HT}$ receptor might remain to be identfied. To address this possibility, RNAi knockdown of predicted, previously uncharacterized, C. elegans biogenic amine receptors (tyra-3, f14d12.6, f16d3.7(ser-5)) was used to screen for additional GPCRs that might be involved in 5-HT-dependent increases in octanol sensitivity in both wild-type and RNAisensitive, $r r f-3$ (pk1269), strains (Simmer et al., 2002). As noted in Figure $4 A, \operatorname{ser}-5(R N A i)$ completely abolished 5-HT-dependent increases in octanol sensitivity in the $r r f-3$, but not wild-type animals. Consistent with these RNAi results, ser-5(tm2654) and ser-5(tm2647) animals exhibited wild-type responses to dilute octanol, but also failed to increase octanol sensitivity in the presence of 5-HT (Fig. 4B). ser-5(tm2654) animals contain a 348 bp deletion in exon 4 resulting in a deletion of a portion of TM5, the third intracellular loop and one-half of TM6. ser-5(tm2647) animals contain a 219 bp deletion in exon 4 , resulting in a deletion of a portion of TM4, the third extracellular loop, TM5 and a portion of the third intracellular loop. Therefore, ser-5(tm2654) and ser-5(tm2647) are predicted null alleles, because TMs 5 and 6 are required for ligand-binding (Strader et al., 1989; Choudhary et al., 1993; Almaula et al., 1996; Roth et al., 1997) and G-protein coupling (Moro et al., 1993).

As anticipated, food- and 5-HT-dependent increases in responses to dilute octanol could be restored in ser-5 null animals by the expression of a full-length ser-5 transgene (Fig. $4 \mathrm{~B}$ ). In addition, 5-HT sensitivity in ser-5 null animals also could be rescued with a sra-6p::ser-5 transgene whose expression is confined primarily to the ASHs, suggesting that SER-5 may function directly in the ASHs (Fig. 4B) (Troemel et al., 1995). Our analysis of ser-5 expression from ser-5p::gfp transcriptional and translational fusions agrees with data reported previously, i.e., fluorescence was observed in vulval and body wall muscle, as well as a number of neurons in the head and tail (Fig. 5C) (Wormbase.org; Carre-Pierrat et al., 2006). GFP fluorescence also was observed in the AWB sensory neurons, and more faintly and variably in the ASHs (Fig. 5C). Low levels of SER-5 expression in the ASHs may be sufficient to confer 5 -HT sensitivity and/or, alternatively, 
A.

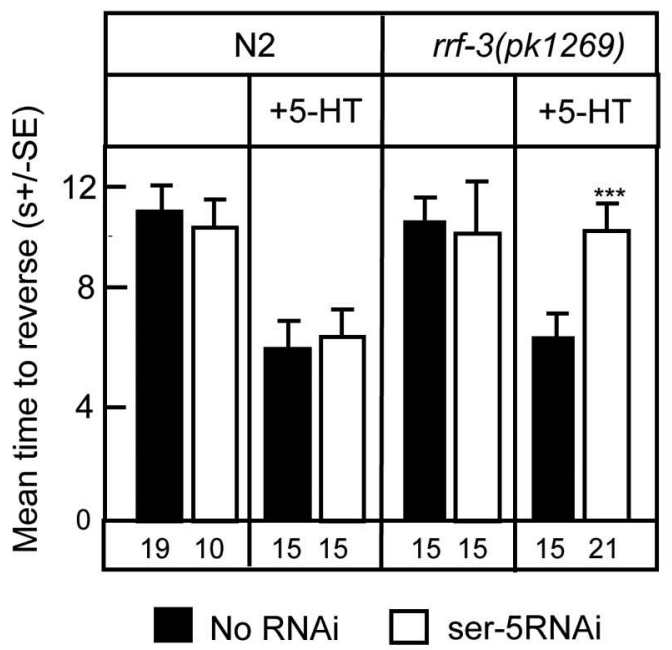

B.

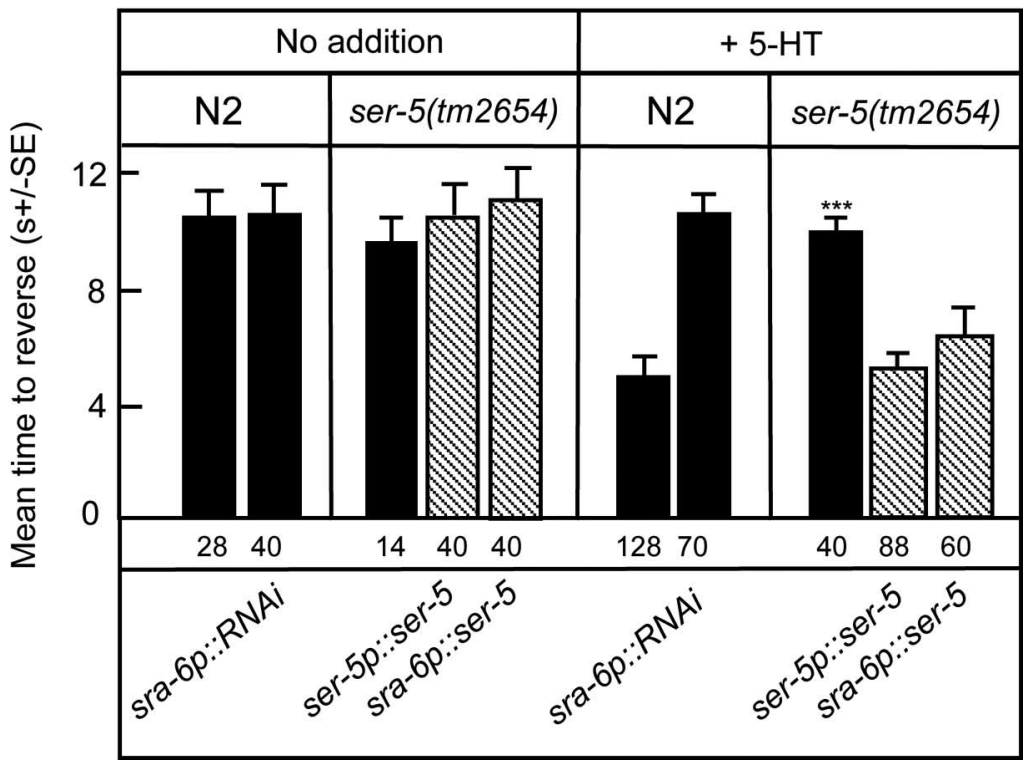

Figure 4. SER-5 is essential for 5-HT-dependent increases in sensitivity to dilute octanol. $\boldsymbol{A}$, Effect of ser-5 RNAi on sensitivity to dilute ( $30 \%$ ) 0 octanol in both wild-type and $r$ rf-3( $p k 1269$ ) animals. $\boldsymbol{B}$, Wild-type and ser-5 null animals were examined for aversive responses to dilute octanol in the presence or absence of 5-HT (4 mm), as described in Materials and Methods. In addition, ser-5 expression in the ASH sensory neurons was knocked down in wild-type animals (sra-6p::ser-5(RNAi) animals). Data are presented as mean \pm SE and analyzed by two-tailed Student's $t$ test. ${ }^{* * *} p<$ 0.001 , significantly different from wild-type or rrf-3(pk1269) animals under identical test conditions. The number of trials is indicated under the bars and results from rescued ser-5 null animals are indicated by hatched bars.

SER-5 might be acting at some other site in the serotonergic circuit. Therefore, to confirm a role for SER-5 directly in the ASHs we examined the 5-HT sensitivity of aversive responses to dilute octanol in wild-type animals in which SER-5 expression in the ASHs was knocked down by the expression of a sra-6p::ser-5(RNAi) transgene. As predicted, these animals exhibited a wild-type response to dilute octanol, but failed to increase octanol sensitivity in response to 5-HT, as observed above for the two ser-5 null mutants (Fig. $4 \mathrm{~B}$ ). Together, these results suggest that SER-5 functions in the ASHs and increases their responsiveness to dilute octanol. Whether SER-5 increases the sensitivity of the putative octanol receptors, downstream signaling and/or neurotransmitter release remains to be determined.

No ser-5 ESTs are present in the database and during the generation of a full-length ser- 5 cDNA for expression studies using $5^{\prime}$ and $3^{\prime}$ rapid amplification of cDNA ends (RACE), we observed that the predicted ser-5 intron/exon splicing pattern and ser-5 cDNA differed significantly from that published in Wormbase.org (Hapiak et al., 2009). As suggested from the behavioral analyses described above, the new predicted SER-5 amino acid sequence is most closely related to mammalian $5-\mathrm{HT}_{6}$ receptors (Fig. 5A). For example, SER-5 was 35\% identical to the human $5-\mathrm{HT}_{6}$ receptor within predicted transmembrane domains involved in ligand binding. In addition, both SER-5 and $5-\mathrm{HT}_{6}$ have short third intracellular loops (67 and 60 aa, respectively) with $\mathrm{BBXXB}_{296}$ (where $\mathrm{B}$ is a basic amino acid) motifs at the $\mathrm{C}$ termini of the loop that in other GPCRs can confer constitutive activity and a conserved PLRYK ${ }_{169}$ motif in the second intracellular loop that is not found in any other 5-HT receptors. Finally, the genes encoding the mammalian $5-\mathrm{HT}_{6}$ receptors contain two introns within sequence encoding the putative third intracellular and third extracellular loops. The positions of both introns are conserved in ser-5 (Hapiak et al., 2009). Interestingly, all of the previously characterized 5-HT receptors clustered ac- cording to their documented G-protein coupling, regardless of phylogenetic origin, validating the utility of the tree and supporting the identification of SER-5 as a G $\alpha_{\mathrm{s}}$-coupled 5-HT receptor (Fig. 5B). Although this tree was generated using truncated amino acid sequences with the more variable $\mathrm{N}$ and $\mathrm{C}$ termini and third intracellular loops deleted, similar results were obtained using the full-length receptors (data not shown). Interestingly, during lethargus mutations that increase $\mathrm{G} \alpha_{\mathrm{s}}$ signaling dramatically increase aversive responses to dilute octanol in the absence of food or exogenous 5-HT (Raizen et al., 2008). We have observed similar effects in wild-type animals (G. P. Harris, unpublished observation). It will be important to determine whether SER-5 and/or the ASH sensory neurons are essential for this $\mathrm{G} \alpha_{\mathrm{s}}$-mediated increase in octanol sensitivity or lethargus.

The serotonergic receptors and pathways modulating ASHmediated aversive behaviors overlap with those modulating the overall rate of spontaneous reversals

The spontaneous initiation of backward locomotion in C. elegans is regulated by the presence of food and 5-HT (Tsalik and Hobert, 2003, Wakabayashi et al., 2004; Gray et al., 2005; Dernovici et al., 2007). For example, in wild-type animals, 5-HT decreases the rate of spontaneous reversal, based on the observation that tph1 (mg280) null animals that lack detectable 5-HT and animals in which the serotonergic neurons have been ablated exhibit a markedly decreased duration of forward movement, i.e., they reverse more frequently (Wakabayashi et al., 2004). In contrast, 5-HT markedly decreases the time taken to reverse in response to dilute octanol (Chao et al., 2004; present study). Therefore, to better understand these two potentially conflicting observations, we examined the rate of spontaneous reversal in the presence of food and after $1 \mathrm{~min}$ in the absence of food in both the mutant and rescued animals used in the present study (Figs. 2, 3). As noted by others, wild-type animals reverse more frequently im- 
A.

agagaagagtggcaacattagaaacatcggaccatcaccgtcatcgccgtcMFQVSDGDGGVVEEVDYSKIAKLAGISTGNESDVVSEMCKEQ LRIILREKLRTGEQLPPAVLVLLCIPAVIIILLTIFGNLLVLFFKARVGRTNTTLLVWNLGLTDFLVGVIVLPMGAVYLIYRKWIFG RFLCRLWVAA VTFCTCSVVTICVISVDRYLAVTRPLRYKSIVTKTKVISVMTIIWIFSSSILLTTVRWEQPECYDDSLCFVGN EIRHLAH VVFAFFLPASVTLTLYWRIYKLARNRQKALDRGFLMILGSNMNFLTNTLSQQQT TLRVHFGKTNGMVEHQRRV LRTHERIAKTLGVVSCSFLFC LPF GLYLTNYKCSGCVPPIAIDVAS LG CNSMLNPIIYSFTVKEFKRSAFRLVVPIWQF VNRCLPFVPAPPDNILQRIARHVHRHKEKEMQTRHRSFEMSSNKNGMLTTKVRSKRRQTEPNVVGLITPDHKLQTVAStaa ccgaatctcatctcatcatctcttctctttcctcttcctcggacgtgttcatcttcccatgtcataattaatttgtttcctttttactgatccggtgtgatcgatattggagaagaaactcaat ttttgtatataaaacatctcatcttct

B.

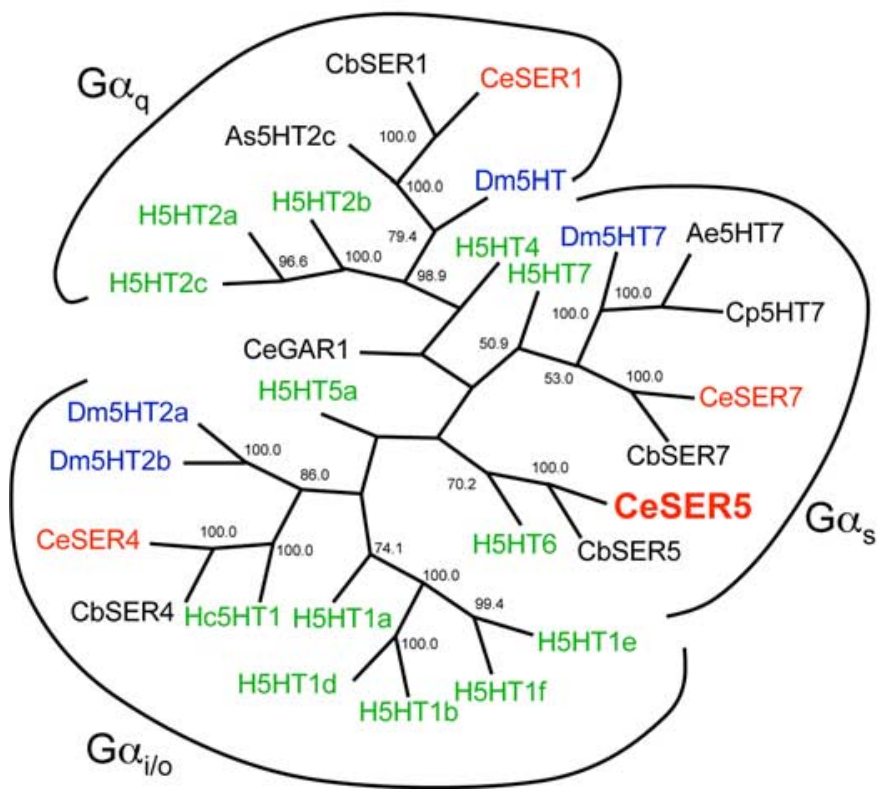

C.
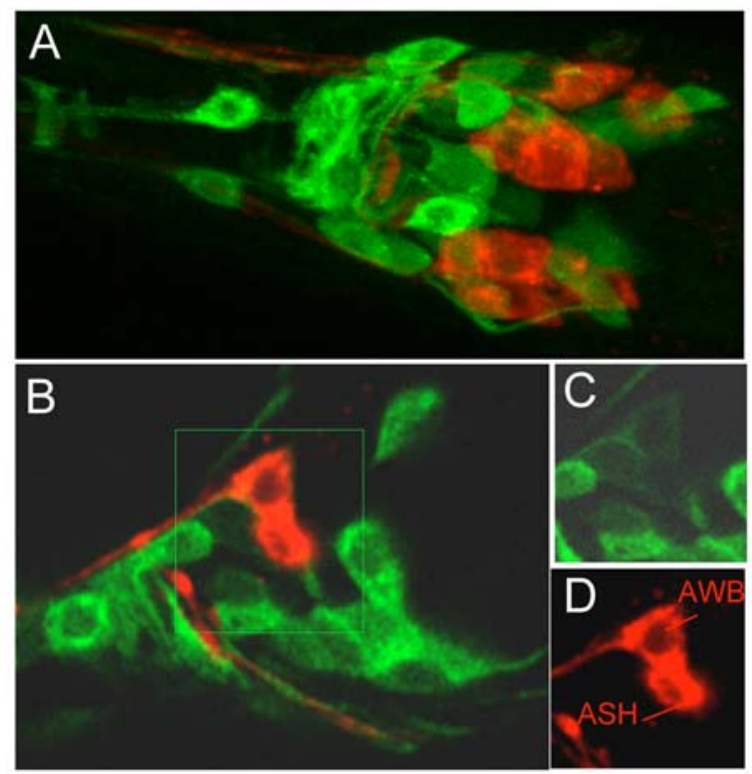

Figure 5. SER-5 is most identical to invertebrate/mammalian 5-HT receptors and is expressed in both neurons and muscle. $\boldsymbol{A}$, Predicted amino acid sequence of SER-5. The full-length ser-5 $\mathrm{CDNA}$ sequence was generated by $5^{\prime}$ and 3' RACE as described in Materials and Methods. Putative transmembrane regions are shaded in gray. Residues potentially involved in BA binding are in white, and G-protein-coupling residues are underlined (Strader et al., 1989; Choudhary et al., 1993; Moro et al., 1993; Barak et al., 1994; Almaula et al., 1996; Roth et al., 1997). Potential PKA and PKC phosphorylation sites were identified using ScanProsite, and are indicated in bold and gray, respectively (Gattiker et al., 2002). Sequestration and desensitization sites are bold and underlined (Barak et al., 1994). B, Unrooted phylogenetic tree of vertebrate and invertebrate 5 -HT receptors. Sequences were modified to remove hypervariable regions by the deletion of the $\mathrm{N}$ termini 3 aa before the first predicted transmembrane domains, the third intracellular loops, 8 aa after and 6 aa before predicted transmembrane domains 5 and 6 , respectively, and the $C$ termini, 12 aa after predicted transmembrane domain 7. Annotated sequences were initially aligned using MegAlign in DNAStar with ClustalW, and using parameters defined in Materials and Methods and fine-tuned by hand. Bootstrapping was undertaken in DNAStar (1000 replicates with random seed). Homo sapiens (H) in green: H5HT1a (CAA40962), H5HT1b (P28222), H5HT1d (P28221), H5HT1e (CAA77558), H5HT1f (AAA36605), H5HT2a (CAA40963), H5HT2b (CAA54513), H5HT2c (AAF35842), H5HT4 (Q13639), H5HT5a (CAA57168), H5HT6 (AAA92622), Hs5HT7 (CAH69965), Caenorhabditis elegans (Ce) in red: CeSER1 (NP_001024728), CeSER4 (NP_497452), CeSER7 (NP_741730), CeSER5. [SER-5 is a potentially new 5-HT receptor in C. elegans, based on new sequence information on ser-5 (based on 5 ' and 3' RACE) (Hapiak et al., 2009) and also based on tw0 5-HT-dependent behavioral phenotypes, egg laying and octanol avoidance (Hapiak et al., 2009; Harris et al., 2009).] Caenorhabditis briggsae (Cb): CbSER-1 (CAE69959), CbSER4 (CAE69091), CbSER7 (CAE58847), CbSER5 (CAE60436), Aedes egyptii (Ae): Ae5HT7 (AAG49292), Drosophila melanogaster (Dm) in blue: Dm5HT7 (NP_524599), Dm5HT2a (NP_725849), Dm5HT2b (CAA77571), Dm5HT (NP_730859), Hemonchus contortus (Hc): Hc5HT1e (AA045883), Ascaris suum (As): As5HT-2c (AAC78396). C, GFP fluorescence from a full-length ser-5p:::ser-5::gfp transgene that includes sequence coding for GFP inserted into the predicted C terminus of the receptor. $\mathbf{C A}$, Merge of GFP fluorescence and 1, ' $^{\prime}$-dioctadecyl-3,3,3' $3^{\prime}$ tetramethylindodicarbocyanine perchlorate (DiD, a lipophilic dye) staining in the nerve ring. $\boldsymbol{C} \boldsymbol{B}$, Single Z-section of GFP/DiD merge in the nerve ring. $\boldsymbol{C}$, $\boldsymbol{C} \boldsymbol{D}$, Insets from $\boldsymbol{C} \boldsymbol{B}$ with GFP fluorescence $(C C)$ and DiD staining $(C D)$.

mediately off food compared with on food (Figs. 2, 3) (Tsalik and Hobert, 2003). This is also true for ser-1, $\bmod -1, \operatorname{ser}-5$ and ser-4; mod-1;ser-7 ser-1 null animals (Fig. 2, 3; data not shown, Dernovici et al., 2007). However, ser-1 or mod-1 null animals reverse significantly more frequently than wild-type animals off food (Figs. $2 B, 3 B$ ) and significantly less frequently than wild-type animals on food (Figs. $2 C, 3 C$ ). The decreased reversal frequencies of the ser- 1 and mod-1 null animals on food contrast sharply with those of tph-1 null animals that lack 5-HT or ser-4;mod-1; ser- 7 ser- 1 animals that lack most serotonergic signaling, i.e., both tph-1 and ser-4;mod-1;ser-7 ser-1 null animals reverse significantly more frequently than wild-type animals on food.

As noted above, the expression of mod-1 in either the AIYs $(t t x-3 p)$, AIBs/AIZs $(o d r 2(2 b) p)$ or AIBs $(n p r-9 p)$ interneurons was sufficient to rescue the 5-HT-dependent stimulation of aversive responses to dilute octanol in mod-1 null animals, suggesting that the AIBs and probably AIYs play essential roles in modulating the 5-HT sensitivity to dilute octanol (Fig. 3, Table 1). In contrast, as shown in Figure 2, the expression of mod-1 in the AIBs or AIBs/AIZs of mod-1 null animals on food or in the AIBs of mod-1 null animals off food did not restore the rate of spontaneous reversals to wild-type levels. The rate of spontaneous reversal was only rescued to wild-type levels in animals expressing mod- 1 in the AIYs on food or the AIYs and AIBs/AIZs off food (Fig. 2B,C). Together these results suggest that MOD-1 plays different roles in modulating the rate of spontaneous reversal and 
Table 1. Identification of neurons expressing MOD-1 that are involved in food/5-HT modulation of spontaneous reversals and aversive responses to dilute octanol

\begin{tabular}{|c|c|c|c|c|}
\hline \multirow[b]{3}{*}{ Strain } & \multirow[b]{3}{*}{ Phenotype } & \multicolumn{3}{|l|}{ Reversals } \\
\hline & & \multirow[b]{2}{*}{0 ctanol $+5-\mathrm{HT}$} & \multicolumn{2}{|c|}{ Spontaneous } \\
\hline & & & Onfood & Off food \\
\hline$t t x-3$ null & No AIY & 0 & + & + \\
\hline mod-1 null & No MOD-1 & - & - & + \\
\hline \multicolumn{5}{|l|}{ mod-1 rescue } \\
\hline$t t x-3 p$ & AIY & $\mathrm{R}$ & $\mathrm{R}$ & $\mathrm{R}$ \\
\hline$o d r-2 b p$ & AIB/AIZ & R & NR & $\mathrm{R}$ \\
\hline$n p r-9 p$ & AIB & $\mathrm{R}$ & NR & NR \\
\hline
\end{tabular}

All null animals and rescued strains were examined for spontaneous reversals and aversive responses to dilute octanol (30\%) as outlined in Figure 3 . Responses are indicated as greater than $(+)$, less than $(-)$, or the same as (0) wild-type animals. R, Rescued; NR, not rescued in mod- 7 null animals.

ASH-mediated aversive responses and suggest a potential role for the AIZ interneurons in modulating the rate of spontaneous reversal immediately off food.

The ser- 1 transgene used to rescue the 5-HT sensitivity of aversive responses to dilute octanol in ser-1 null animals also restored the rate of spontaneous reversal to wild-type levels both on and immediately off food (Fig. $3 B, C$ ). This ser-1 transgene appears to be expressed in the RIA and number of ring motor/ interneurons (Xiao et al., 2006; Dernovici et al., 2007). Interestingly, animals lacking RIAs ( $g l r-3 p:: I C E)$ or with reduced SER-1 expression in the RIAs ( $g l r-3 p:: s e r-1(R N A i))$ failed to increase aversive responses to dilute octanol in presence of 5-HT (Fig. 3A), but still exhibited wild-type rates of spontaneous reversal both on and immediately off food (Fig. 3 B, C) (Tsalik and Hobert, 2003). These results highlight the importance of the RIAs in the serotonergic sensitization of aversive responses to dilute octanol and suggest that other neurons, possibly the ring motor neurons, are responsible for SER-1 mediated modulation of spontaneous reversal.

Together, these results highlight the complexity of serotonergic signaling in the modulation of reversal responses and suggest that ser-1 and mod-1 inhibit reversal off food and stimulate reversal on food, in agreement with their proposed stimulatory roles in mediating the food- and 5-HT-dependent increases in aversive responses to dilute octanol observed above.

\section{Discussion}

In the present study, we have identified three amine receptors that are each essential for 5-HT-dependent increases in aversive responses to dilute octanol. Off food, wild-type, and 5-HT receptor mutants respond identically to dilute octanol. In contrast, on food or in the presence of 5-HT, wild-type animals respond more rapidly and this increased aversive response is completely abolished in animals lacking either SER-5, MOD-1, or SER-1. Interestingly, no intermediate responses were observed; each of the receptors appear to be essential for 5-HT sensitization, suggesting that each plays a different, but critical role in the process.

\section{Serotonergic signaling enhances responses to dilute octanol at} multiple levels within the ASH-mediated locomotory circuit

Each of the receptors that are essential for the serotonergic sensitization of aversive responses to dilute octanol operate at different levels within the ASH-mediated locomotory circuit: SER-5 in ASH sensory neurons, MOD-1 in the AIB interneurons that receive direct input from the ASHs, and SER-1 in RIA/ring motor neuron pathway that innervates head muscle (Fig. 6). Based on the wiring diagram of the C. elegans nervous system, we predict that SER-5 increases the responsiveness of the ASHs to initiate backward locomotion, MOD-1 modulates signaling to the command interneurons through the AIBs to inhibit forward locomotion, and SER-1 modulates signaling to head muscle (Fig. 6). For example, 5-HT stimulated responses in ser-1 animals could be rescued with a ser-1p::ser-1::gfp transgene that is expressed in the RIAs and the RMD ring motor neurons that innervate head muscle (Xiao et al., 2006; Dernovici et al., 2007). Ablation of the RIAs or the knockdown of ser-1 expression in the RIAs has no effect on the rate of spontaneous reversal or octanol sensitivity off food, but abolishes 5-HT-dependent increases in sensitivity to dilute octanol, suggesting that the sensitization of head muscle may be essential for stimulating aversive responses, especially when the ASH is less than maximally stimulated.

\section{Food/serotonin activates SER-5 in the ASH sensory neurons}

Serotonin (5-HT) is one of the "food is at hand" signals in $C$. elegans and regulates most aspects of $C$. elegans behavior. Four $C$. elegans 5-HT receptors have been identified previously, three G-protein-coupled receptors, SER-1, SER-4, and SER-7 that appear to signal through $\mathrm{G} \alpha_{\mathrm{q}}, \mathrm{G} \alpha_{\mathrm{o}}$, and $\mathrm{G} \alpha$, respectively, and a novel 5-HT-gated chloride channel, MOD-1. In addition, we have identified a fifth putative 5-HT receptor, SER-5. Previously, the roles of these receptors in pharyngeal pumping (Hobson et al., 2003), egg-laying (Hobson et al., 2003; Carnell et al., 2005; Dempsey et al., 2005; Xiao et al., 2006), the enhanced slowing response (Ranganathan et al., 2000), and aversive learning (Zhang et al., 2005) have been characterized. In addition, 5-HT plays a role in modulating locomotory transitions associated with nutritional status (Fujiwara et al., 2002; Tsalik and Hobert, 2003; Gray et al., 2005). However, a thorough understanding of the anatomy and overall modulation of serotonergic signaling is still in its infancy and few studies have localized the 5-HT receptors involved in mediating individual behaviors.

The role of feeding status and 5-HT in the modulation of octanol avoidance has been demonstrated previously (Chao et al., 2004). The ASH sensory neurons are both necessary and sufficient for responses to dilute octanol and food/5-HT increases these responses. Similarly, nose touch, another ASH-mediated behavior, elicits 5-HT-dependent $\mathrm{Ca}^{2+}$ transients in the ASHs, even in unc-13 animals, strongly suggesting that 5-HT alters the sensitivity of the ASHs directly (Hilliard et al., 2005). Indeed, we have identified a putative 5-HT receptor, SER-5, that functions directly in the ASHs. SER- 5 is most closely related to mammalian $5-\mathrm{HT}_{6}$ receptors. $5-\mathrm{HT}_{6}$ receptors are abundantly expressed in olfactory tubercles, although no studies have examined their role in mammalian nociception (Woolley et al., 2004; Mitchell and Neumaier, 2005). Because the 5-HT specificity of SER-5 has not been demonstrated through ligand-binding studies, it is possible that an additional, as yet unidentified, 5-HT receptor is involved in the release of another neuromodulator that then activates SER-5. We consider this a remote possibility, given the close alignment of the new SER-5 sequence with other 5-HT receptors and the identification of a second 5-HT stimulated behavior that is dependent on SER-5, i.e., 5-HT-dependent increases in egglaying in ser-4;mod-1;ser-7 ser-1 null animals (Hapiak et al., 2009). Attempts to express SER-5 in mammalian cells with the addition of signal sequences or temperature shock, as we have described for other C. elegans GPCRs, have been unsuccessful (Rex and Komuniecki, 2002; Hobson et al., 2003). Indeed, the direct characterization of SER-5 may be problematic in the absence of a nematode cell line. For example, the heterologous expression of many olfactory and pheromone GPCRs that are ex- 
pressed in sensory neurons is poor and may require accessory proteins (Loconto et al., 2003).

Both the AIB and potentially the AIY interneurons are involved in the MOD1-dependent sensitization of ASHmediated aversive responses

The ASH sensory neurons innervate the AVA/AVB/AVD command interneurons that likely function as a "bistable switch" to regulate forward/backward locomotion and reversal (Chalfie et al., 1985; Zheng et al., 1999; Brockie et al., 2001; Tsalik and Hobert, 2003; Gray et al., 2005). In addition, the ASHs synapse on the AIA, AIB and RIA interneurons, but not on the motorneurons that directly innervate body wall muscle. 5-HT stimulated responses to dilute octanol can be rescued in $\bmod -1$ animals by expression of mod-1 transgenes in the AIBs or AIYs ( $n p r-9 p$ or $t t x-3 p)$. Similarly, the expression of $o d r-2(2 b) p:: \bmod -1$ (AIBs/AIZs) or $t t x-3 p::$ mod-1 transgenes rescued aversive learning in mod-1 animals (Zhang et al., 2005). The AIBs are innervated by the ASHs and are prominent postsynaptic partners of the AIAs. Because the expression of mod- 1 in the AIBs alone rescued 5-HT-dependent increases in octanol sensitivity, these results suggest that the AIBs play a key role in mediating the effects of MOD-1 signaling on octanol sensitivity (White et al., 1986).

The AIYs also appear to be involved in mod-1-mediated aversive behaviors, although they are not directly innervated by the ASHs. In fact, many sensory neurons mediate locomotory behaviors through the AIYs (Ryu and Samuel, 2002; Tsalik and Hobert, 2003; Zariwala et al., 2003). For example, $t t x-3$ mutants, in which the AIYs fail to develop, do not modulate reversal frequency in response to ASE-mediated gustatory or AWA/AWC-mediated olfactory cues (Tsalik and Hobert, 2003). In contrast, in the present study, ttx-3 null animals exhibited wild-type aversive responses to dilute octanol in both the presence and absence of 5-HT. However, mod-1 encodes a 5-HT-gated chloride channel that would presumably inhibit AIY function. Therefore, as observed in the present study, aversive responses to dilute octanol on food would be predicted to be stimulated in both wild-type animals without AIYs, as observed in ttx-3 animals or in animals in which the AIYs are inhibited by MOD-1, as in the $t t x-3 p:: \bmod -1$ rescued animals. It is worth noting that the expression of an inhibitory $\mathrm{Cl}^{-}$channel has the potential to silence neurons and could affect behavior, even if these neurons are not normally involved in the behavior. However, based on expression of mod-1 and neuron-specific rescue of mod-1 animals, these results suggest that both the AIBs and AIYs are involved in the MOD-1-dependent modulation of octanol sensitivity in the presence of food or 5-HT.

Both the AIBs and AIYs also modulate spontaneous reversal (Tsalik and Hobert, 2003; Gray et al., 2005). For example, AIB ablation prevents the stimulation of long reversals and $\omega$ turns when animals are removed from food, suggesting that the AIBs increase the probability of reversal during area restricted search (Gray et al., 2005). Similarly, ablation of the AIYs increases the frequency of spontaneous reversals both on and off food and roles have been proposed for the AIYs in modulating reversals associated with other behaviors (Mori and Ohshima, 1997; Tsalik and Hobert, 2003; Gray et al., 2005). The AIBs and AIYs appear to act, at least in part, in parallel, based on the observation that their roles in the modulation of locomotory behavior appear to be additive (Gray et al., 2005). In the present study, spontaneous reversal was similarly stimulated in mod-1 and ttx-3 animals off food. In contrast, reversal frequency was inhibited in mod-1 animals and stimulated in $t t x-3$ animals on food. Together with the data on octanol sensitivity described above, these observations highlight the different, but overlapping, roles played by serotonergic signaling in the modulation of spontaneous reversals or ASH-mediated aversive responses.

In summary, our observations suggest that the serotonergic modulation of aversive responses involves sensitization at multiple levels within the ASH-mediated circuit that controls reversal/ backward locomotion. In addition, they highlight the advantages of the C. elegans model in dissecting the complex aminergic modulation of olfactory behaviors. 


\section{References}

Alkema MJ, Hunter-Ensor M, Ringstad N, Horvitz HR (2005) Tyramine functions independently of octopamine in the Caenorhabditis nervous system. Neuron 46:247-260.

Almaula N, Ebersole BJ, Zhang D, Weinstein H, Sealfon S (1996) Mapping the binding site pocket of the serotonin 5-hydroxytryptamine2A receptor. Ser3.36(159) provides a second interaction site for the protonated amine of serotonin but not of lysergic acid diethylamide or bufotenin. J Biol Chem 271:14672-14675.

Barak LS, Tiberi M, Freedman NJ, Kwatra MM, Lefkowitz RJ, Caron MG (1994) A highly conserved tyrosine residue in G-protein coupled receptors is required for agonist-mediated $\beta_{2}$-adrenergic receptor sequestration. J Biol Chem 269:2790-2795.

Bargmann CI, Hartwieg E, Horvitz HR (1993) Odorant-selective genes and neurons mediate olfaction in C. elegans. Cell 74:515-527.

Bendena WG, Boudreau JR, Papanicolaou T, Maltby M, Tobe SS, Chin-Sang ID (2008) A Caenorhabditis elegans allatostatin/galanin-like receptor NPR-9 inhibits local search behavior in response to feeding cues. Proc Natl Acad Sci U S A 105:1339-1342.

Brenner S (1974) The genetics of Caenorhabditis elegans. Genetics 77:71-94.

Brockie PJ, Mellem JE, Hills T, Madsen DM, Maricq AV (2001) The C. elegans glutamate receptor subunit NMR-1 is required for slow NMDAactivated currents that regulate reversal frequency during locomotion. Neuron 31:617-630.

Carnell L, Illi J, Hong SW, McIntire SL (2005) The G-protein-coupled serotonin receptor SER-1 regulates egg laying and male mating behaviors in Caenorhabditis elegans. J Neurosci 25:10671-10681.

Carre-Pierrat M, Baillie D, Johnsen R, Hyde R, Hart A, Granger L, Ségalat L (2006) Characterization of the Caenorhabditis elegans $\mathrm{G}$ protein-coupled serotonin receptors. Invert Neurosci 6:189-205.

Chalfie M, Sulston JE, White JG, Southgate E, Thomson JN, Brenner S (1985) The neural circuit for touch sensitivity in Caenorhabditis elegans. J Neurosci 5:956-964.

Chao MY, Komatsu H, Fukuto HS, Dionne HM, Hart AC (2004) Feeding status and serotonin rapidly and reversibly modulate a Caenorhabditis elegans chemosensory circuit. Proc Natl Acad Sci U S A 101:15512-15517.

Chao MY, Larkins-Ford J, Tucey TM, Hart AC (2005) LIN-12 Notch functions in the adult nervous system of C. elegans. BMC Neurosci 6:45.

Chou JH, Bargmann CI, Sengupta P (2001) The Caenorhabditis elegans $o d r-2$ gene encodes a novel Ly-6-related protein required for olfaction. Genetics 157:211-224.

Choudhary MS, Craigo S, Roth BL (1993) A single point mutation (Phe340 $\rightarrow$ Leu340) of a conserved phenylalanine abolishes 4-[125I]iodo(2,5-dimethoxy)phenylisopropylamine and $[3 \mathrm{H}]$ mesulergine but not $[3 \mathrm{H}]$ ketanserin binding to 5-hydroxytryptamine2 receptors. Mol Pharmacol 43:755-761.

de Bono M, Maricq AV (2005) Neuronal substrates of complex behaviors in C. elegans. Annu Rev Neurosci 28:451-501.

Dempsey CM, Mackenzie SM, Gargus A, Blanco G, Sze JY (2005) Serotonin (5HT), fluoxetine, imipramine and dopamine target distinct 5HT receptor signaling to modulate Caenorhabditis elegans egg-laying behavior. Genetics 169:1425-1436.

Dernovici S, Starc T, Dent JA, Ribeiro P (2006) The serotonin receptor SER-1 (5HT2ce) contributes to the regulation of locomotion in Caenorhabditis elegans. Dev Neurobiol 67:189-204.

Esposito G, Di Schiavi E, Bergamasco C, Bazzicalupo P (2007) Efficient and cell specific knock-down of gene function in targeted C. elegans neurons. Gene 395:170-176.

Fujiwara M, Sengupta P, McIntire SL (2002) Regulation of body size and behavioral state of $C$. elegans by sensory perception and EGL-4 cGMPdependent protein kinase. Neuron 36:1091-1102.

Gattiker A, Gasteiger E, Bairoch A (2002) ScaProsite: a reference implementation of a PROSITE scanning tool. Appl Bioinformatics 1:107-108.

Gray JM, Hill JJ, Bargmann CI (2005) A circuit for navigation in Caenorhabditis elegans. Proc Natl Acad Sci U S A 102:3184-3191.

Hamdan FF, Ungrin MD, Abramovitz M, Ribeiro P (1999) Characterization of a novel serotonin receptor from Caenorhabditis elegans: cloning and expression of two splice variants. J Neurochem 72:1372-1383.

Hapiak VM, Hobson RJ, Hughes L, Smith K, Harris G, Condon C, Komuniecki P, Komuniecki RW (2009) Dual excitatory and inhibitory serotonergic inputs modulate egg laying in Caenorhabditis elegans. Genetics 181:153-163.
Heisler LK, Zhou L, Bajwa P, Hsu J, Tecott LH (2007) Serotonin 5-HT(2c) receptors regulate anxiety-like behavior. Genes Brain Behav 6:491-496.

Herman RK, Hedgecock EM (1990) Limitations of the size of the vulval primordium of Caenorhabditis elegans by lin-15 expression in surrounding hyopodermis. Nature 348:169-171.

Hilliard MA, Apicella AJ, Kerr R, Suzuki H, Bazzicalupo P, Schafer WR (2005) In vivo imaging of C. elegans ASH neurons: cellular response and adaptation to chemical repellents. EMBO J 24:63-72.

Hobert O (2002) PCR fusion-based approach to create reporter gene constructs for expression analysis in transgenic C. elegans. Biotechniques $32: 728-730$.

Hobert O, Mori I, Yamashita Y, Honda H, Ohshima Y, Liu Y, Ruvkun G (1997) Regulation of interneuron function in the C. elegans thermoregulatory pathway by the ttx-3 LIM homeobox gene. Neuron 19:345-357.

Hobson RJ, Geng J, Gray AD, Komuniecki RW (2003) SER-7b, a constitutively active G $\alpha$ coupled 5-HT7-like receptor expressed in the Caenorhabditis elegans M4 pharyngeal motorneuron. J Neurochem 87:22-29.

Horvitz HR, Chalfie M, Trent C, Sulston JE, Evans PD (1982) Serotonin and octopamine in the nematode C. elegans. Science 216:1012-1014.

Huang X, Xiao H, Rex EB, Hobson RJ, Messer WS, Komuniecki PR, Komuniecki RW (2002) Functional characterization of alternatively spliced $5-\mathrm{HT}_{2}$ isoforms from the pharynx and muscle of the parasitic nematode, Ascaris suum. J Neurochem 83:249-258.

Kamath RS, Ahringer J (2003) Genome-wide RNAi screening in C. elegans. Methods 30:313-321.

Kramer JM, French RP, Park EC, Johnson JJ (1990) The Caenorhabditis elegans rol-6 gene, which interacts with the sqt-1 collagen gene to determine organismal morphology, encodes a collagen. Mol Cell Biol 10:2081-2089.

Loconto J, Papes F, Chang E, Stowers L, Jones EP, Takada T, Kumánovics A, Fischer Lindahl K, Dulac C (2003) Functional expression of murine $\mathrm{V} 2 \mathrm{R}$ pheromone receptors involves selective association with the M10 and M1 families of MHC class Ib molecules. Cell 112:607-618.

Lucki I (1998) The spectrum of behaviors influenced by serotonin. Biol Psychiatry 44:151-162.

Mello C, Fire A (1995) DNA transformation. Methods Cell Biol 48:451-482.

Mitchell ES, Neumaier JF (2005) 5- $\mathrm{HT}_{6}$ receptors: a novel target for cognitive enhancement. Pharmacol Ther 108:320-333.

Mori I, Ohshima Y (1997) Molecular neurogenetics of chemotaxis and thermotaxis in the nematode Caenorhabditis elegans. BioEssays 19:1055-1064.

Moro O, Lameh J, Högger P, Sadée W (1993) Hydrophobic amino acids in the i2 loop plays a key role in receptor G-protein coupling. J Biol Chem 268:22273-22276.

Niacaris T, Avery L (2003) Serotonin regulates repolarization of the C. elegans pharyngeal muscle. J Exp Biol 206:223-231.

Olde B, McCombie WR (1997) Molecular cloning and functional expression of a serotonin receptor from Caenorhabditis elegans. J Mol Neurosci 8:53-62.

Pierce-Shimomura JT, Morse TM, Lockery SR (1999) The fundamental role of pirouettes in C. elegans chemotaxis. J Neurosci 19:9557-9569.

Ranganathan R, Cannon SC, Horvitz HR (2000) MOD-1 is a serotoningated chloride channel that modulates locomotory behavior in C. elegans. Nature 408:470-475.

Raizen DM, Zimmerman JE, Maycock MH, Ta UD, You YJ, Sundaram MV, Pack AI (2008) Lethargus is a Caenorhabditis elegans sleep like state. Nature 451:569-572.

Rex E, Komuniecki RW (2002) Characterization of a tyramine receptor from Caenorhabditis elegans. J Neurochem 82:1352-1359.

Roth BL, Shoham M, Choudhary MS, Khan N (1997) Identification of conserved aromatic residues essential for agonist binding and second messenger production at 5-hydroxytryptamine $2 \mathrm{~A}$ receptors. Mol Pharmacol $52: 259-266$.

Ryu WS, Samuel AD (2002) Thermotaxis in Caenorhabditis elegans analyzed by measuring responses to defined thermal stimuli. J Neurosci 22:5727-5733.

Sawin ER, Ranganathan R, Horvitz HR (2000) C. elegans locomotory rate is modulated by the environment through a dopaminergic pathway and by experience through a serotonergic pathway. Neuron 26:619-631.

Ségalat L, Elkes DA, Kaplan JM (1995) Modulation of serotonin-controlled behaviors by Go in Caenorhabditis elegans. Science 267:1648-1651.

Simmer F, Tijsterman M, Parrish S, Koushika SP, Nonet ML, Fire A, Ahringer 
J, Plasterk RH (2002) Loss of the putative RNA-directed RNA polymerase RRF-3 makes C. elegans hypersensitive to RNAi. Curr Biol $12: 1317-1319$.

Strader CD, Candelore MR, Hill WS, Sigal IS, Dixon RA (1989) Identification of two serine residues involved in agonist activation of the betaadrenergic receptor. J Biol Chem 264:13572-13578.

Sulston J, Hodgkin J (1988) Methods. In: The nematode Caenorhabditis elegans (Wood WB, ed), pp 587-606. Cold Spring Harbor, NY: Cold Spring Harbor Laboratory.

Troemel ER, Chou JH, Dwyer ND, Colbert HA, Bargmann CI (1995) Divergent seven transmembrane receptors are candidate chemosensory receptors in C. elegans. Cell 83:207-218.

Troemel ER, Kimmel BE, Bargmann CI (1997) Reprogramming chemotaxis responses: sensory neurons define olfactory preferences in C. elegans. Cell 91:161-169.

Tsalik EL, Hobert O (2003) Functional mapping of neurons that control locomotory behavior in Caenorhabditis elegans. J Neurobiol 56:178-197.

Tsalik EL, Niacaris T, Wenick AS, Pau K, Avery L, Hobert O (2003) LIM homeobox gene-dependent expression of biogenic amine receptors in restricted regions of the C. elegans nervous system. Dev Biol 263:81-102.

Waggoner LE, Zhou GT, Schafer RW, Schafer WR (1998) Control of alternative behavioral states by serotonin in Caenorhabditis elegans. Neuron 21:203-214

Wakabayashi T, Kitagawa I, Shingai R (2004) Neurons regulating the dura- tion of forward movement in Caenorhabditis elegans. Neurosci Res 50:103-111.

Wenick AS, Hobert O (2004) Genomic cis-regulatory architecture and trans-acting regulators of a single interneuron-specific gene battery in $C$. elegans. Dev Cell 6:757-770.

White JG, Southgate E, Thomson JN, Brenner S (1986) The structure of the nervous system of the nematode Caenorhabditis elegans. Philos Trans R Soc Lond B Biol Sci 314:1-340.

Woolley ML, Marsden CA, Fone KC (2004) 5-HT6 receptors. Curr Drug Targets CNS Neurol Disord 3:59-79.

Xiao H, Hapiak VM, Smith KA, Lin L, Hobson RJ, Plenefisch J, Komuniecki RW (2006) SER-1, a Caenorhabditis elegans 5-HT like receptor, and a multi-PDZ domain containing protein (MPZ-1) interact in vulval muscle to facilitate serotonin-stimulated egg laying. Dev Biol 298:379-391.

Zariwala HA, Miller AC, Faumont S, Lockery SR (2003) Step response analysis of thermotaxis in Caenorhabditis elegans. J Neurosci 23:4369-4377.

Zhang Y, Lu H, Bargmann CI (2005) Pathogenic bacteria induce aversive olfactory learning in Caenorhabditis elegans. Nature 438:179-184.

Zhao B, Khare P, Feldman L, Dent JA (2003) Reversal frequency in Caenorhabditis elegans represents an integrated response to the state of the animal and its environment. J Neurosci 23:5319-5328.

Zheng Y, Brockie PJ, Mellem JE, Madsen DM, Maricq AV (1999) Neuronal control of locomotion in C. elegans is modified by a dominant mutation in the GLR-1 ionotropic glutamate receptor. Neuron 24:347-361. 